\title{
The Coherence Definition of Consciousness
}

Christoph von der Malsburg

Institut für Neuroinformatik, Ruhr-Universität Bochum

and

Departments of Computer Science and Biology, University of Southern California, Los Angeles 
Introduction.

I am not sure it isn't a mistake to write about consciousness - too little is my chance of adding anything to what the Great have already said, too thickly the issue overgrown with philosophical argument, and too diverse the viewpoints emphasized by different people. Most discouraging of all, little progress is in sight regarding the qualia issue, the question of how a material thing, our brain, can create the vivid sensations we experience. I feel that essential scientific and technical breakthroughs are required before the consciousness issue can be made to progress substantially. The next major impulse may have to await the advent of artificial organisms we can talk to and that give us the impression they are conscious. It is my belief that this venture of building artificial conscious organisms will not encounter insurmountable barriers (although progress will be difficult), and that once they are here we will have to revise our notions on a basic level, just as biologists had to do so vis-à-vis the vis vitalis issue after the organic chemistry revolution.

I will focus in this essay on a riddle that in my view is central to the consciousness issue: How does the mind or brain create the unity we perceive out of the diversity that we know is there? I contend this is a technical issue, not a philosophical one, although its resolution will have profound philosophical repercussions, and although we have at present little more than the philosophical method to attack it.

A profound revolution has swept across the intellectual world in the last one or two decades as a result of which we are now ready to see the creation of order in complex systems not as the result of extraneous pre-existing plans but of spontaneous interactions of simple agents. In my view this was an essential philosophical adaptation putting us in a much better position now to attack the consciousness issue.

The Unity of Mind.

Our introspective experience is one of unity, of monolithic coherence. This unity characterizes consciousness and awareness, attention, perception and action, our decisions and our will, our personality and self and to a large extent our value judgments. This unity prevails in spite of the diversity of aspects, influences, perceptions, impulses, memories, processes and agents that we find as components of our mind, or, from a different perspective, in spite of the tremendous mass of building elements in our brain - areae, nuclei, nerves, neurons, fibers, synapses, membranes and molecules. Creating the mind's unity out of the brain's diversity, the problem of nervous integration, is now, after the aforementioned revolution, an issue of scientific, technical nature - How does the brain manage to let its myriad elements cooperate to create a coherent functional whole?

A now essentially defunct view sees all of the underlying diversity of the brain essentially as passive material arranged and ordered by a separate perceiving and planning entity, sometimes insisting on an immaterial nature of the mind. Very often this view sees itself tempted to squeeze the unity-creating entity into a tight place - the pineal gland (as did Descartes 1662) or some other small anatomical part of the brain —, maybe just trying to deprive the poor thing of space for internal disagreement, or falling prey to the deeply ingrained prejudice that order is to be created with the help of central control - a center 
being, of course, a separate entity of small extent. It is perhaps just another attempt to understand the mind's unity when identifying consciousness with just one modality, for instance short- term memory (Erdelyi 1974), the ability to enact plans (Shallice 1978), the ability to detect and put away obstacles encountered in unconscious processes (Mandler 1975), language (Sperry 1966) or the self (Minsky 1988). Unfortunately, even single modalities aren't natural, monolithic units, and on closer inspection they become themselves extensive and diverse systems, creating the same riddle as before.

One attempt at the unity-out-of-diversity problem relies on unity of action, on the impossibility for our limbs and muscles to move in more than one direction at a time. This alone wouldn't explain coherent motion flowing from a chaotic brain. It would rather have to be seen as a persistent influence on the brain to weed out self-contradiction and incoherence. Although this certainly is an important factor, the argument still shuns the original question of how the brain creates those coherent states.

Perhaps the most grotesque - although at the same time so strangely compelling - device to deal with the unity problem is the homunculus, the idea of a separate planning and perceiving agent, hidden somewhere in the brain and structured after the model of a coherently acting, deciding and perceiving human. The origin of this compulsion is evidently the basic experience of ours that confronted with diverse stuff before our eyes our mind creates a coherent whole out of it. So rapid and effortless is this process that we have long since come to incorporate it in our basic conception of the world as a primary constituent, as elementary as gravity and air. And the same way it is difficult to discover even the problem of how gravity works, the phenomenon already being deeply ingrained in our mind when we start thinking, it is difficult to recognize as such the problem of how the mind creates order. Consequently man has used this phenomenon of "spontaneous" creation of order by the mind as one of the fundamental metaphors to understand the world: wherever there is order, there must have been a mind to create it. The concept of the homunculus results very naturally from the application of this metaphor to the enigma of creation of order and unity in the mind itself! The infinite regress arising from the question how the homunculus creates his own internal order has been gloated over enough to need more comment.

\section{Consciousness as Degree of Coherence of the Mind.}

Introspectively, we can easily distinguish different degrees of consciousness. Driving your car you are entertaining a conversation with this attractive person. All of a sudden, your passenger asks why you go so fast. You immediately slow down and reply you hadn't been conscious of your driving, paying attention to the conversation. Now you are conscious of it, perhaps feeling like an idiot to take orders from that person, or to have given the impression of being over-excited.

In this example, a large sector of your mind (the one occupied with driving) was first shut off from communication with other modalities and was then suddenly opened to interact with them, so that you now can comment on it, place it in a social context, remember details and draw conclusions. Along similar lines you find states of mind that are more or less fractionated into disconnected activities and distracted by various influences, one 
part of your mind not being able to react to the events in other parts; and other states where all your capacities are highly focused on one point, ready to react should anything important be noticed by any one agent of your mind.

Typical states of high consciousness are those involving intense perceived danger, when we are readying all of our senses and faculties to save us from a threat. Another potent stimulus to raise our level of consciousness is the detection of an obstacle in the performance of a routine task. When thus alerted we start bringing to bear creative infrastructure to help us solve the problem - diagnostic tools, reference to underlying goals and strategies, memories of similar situations and analogous problems. We perceive such states of mind as on a higher level of consciousness (Mandler 1975), albeit in a temporally more drawn-out way than the more acute states of alert.

Observations of this kind lead us to the conclusion that, even in the waking state, we experience mind states of different degree of consciousness, and that the difference is made by the degree of coherence, or order, or readiness to communicate, between parts of the brain. Let us, then, describe a state of highest consciousness as one characterized by global order among all the different active processes going on simultaneously in your mind, by maximal coherence and consistency between the different aspects of and perspectives on the current subject matter, one in which the full mental power you can muster is thrown onto the same issue, one in which only such material is activated as is relevant to the present interest.

The mind in a state of consciousness can be described as an organism composed of subsystems that are causally connected with each other. Each of the subsystems is actively observing what the others are doing and is ready to react to any significant change in them. (The mind as a "society of agents" has been discussed by Minsky (1988) although without reference to the consciousness issue.) These reactions are necessary to maintain coherence across all larger and smaller changes in subject matter as they occur. Sometimes very drastic and global changes of state happen, all subsystems switching to a new topic almost instantly, and it may not be easy or possible to track the change back to a local cause in one of the communicating modalities. In other instances the cause for a change may be obvious, such as when an external event hits our senses, draws the attention of many agents to it and causes them to react.

A perhaps enlightening physical metaphor for the conscious state of the brain is a system of communicating cavity resonators supporting acoustic or electromagnetic oscillations. A globally coupled state could be one in which all the different cavities are phase-locked to each other. A local event would shift the phase or frequency of one of the cavities, and the rest of the system could adjust for it by altering phases and frequencies accordingly. A "subconscious" state would be one in which the system decomposed into subsystems without fixed phase relationships between them, and in a fractionated state like that the oscillations in many cavities would not adjust in response to a local change elsewhere in any consistent way. Another metaphor from physics refers to systems capable of phase transitions. A good example, discussed as such by Toulouse et al. (1986) is a spin glass. Any configuration change in a subsystem of sufficient size can precipitate a more or less 
global phase transition, many other subsystems reacting such as to lead to minimum free energy again.

What is Coherence?

My definition of consciousness as a brain state of a high level of coherence between subsystems requires a definition of the term coherence. One could be tempted to base a definition of mental coherence on insight into the semantic content of the modalities involved and on judgment about mutual relevance of their present state. However, such a definition would be impractical or impossible. In fact, there is reason to believe that it is not necessary to refer explicitly to the semantic content of different modalities and that a simple and quite general definition of coherence can be found.

The essence of coherence is successful collaboration of modalities in solving problems. In order to function properly, the brain needs general mechanisms to diagnose and establish such collaboration. The brain's creation by evolution antedates the generation of many of the specific agents present in modern man's mind. The structure of the mechanisms of organization must therefore be independent of specific subject matters and consequently fairly simple.

Let me speculate a little what those mechanisms could be. When inspecting an object it gets reflected differently in different modalities. One important mechanism for defining coherence might be the associative mechanism, by which the patterns simultaneously appearing in the different modalities are synaptically linked to each other. This mechanism can be very potent if attention is quickly shifted between sub-components of a perceived situation and associations are established between all sets of patterns simultaneously lighting up in different places in the brain. Such coincidences as appear only accidentally will not have lasting effects, whereas reliable cross-predictions of events in different modalities will establish permanent relationships.

This establishment of functional relations between modalities makes sense only for fairly elementary sub-patterns of actual situations. Only these have a chance of recurring, entire situations being unique events. (How appropriate sub-patterns of situations are picked out for storage during learning still is to a large extent unknown.) Confronted with a new situation, all modalities are active with new complex patterns that cannot be related directly to each other. If the situation is, however, decomposed into known elementary patterns, the relationships between the complex patterns in the different modalities can be disambiguated and useful maps between them can be pieced together. The price for this disambiguation and structuring of relationships is narrowly focused attention and the necessity to sequentially scan previously learned sub-patterns.

According to this analysis coherence, and consequently consciousness, are only possible at the expense of restricting scenes to definite interpretations in terms of known subpatterns. This point has been emphasized by Kahneman (1973), and according to Posner (1987) "the key to understanding the nature of conscious attention is its limited capacity" (p. 153). The significance and depth of this connection between coherence and restriction or limitation of substance is far from being fully grasped. 
The Mind as a Historical Process.

According to the coherence definition I am advocating here only those brain states are conscious in which as many modalities as possible are functionally coupled to each other. One important aspect of this coupling is that modalities have to be disposed to react to changes and events taking place in any of the others. This leads to an operational test and a basis for an experimental procedure: create some appropriate and unexpected changes in individual modalities and see whether the others do react in appropriate ways: if they do, that mind is conscious. There are several difficulties with this experiment, not the least of which the danger of altering the subject's state of consciousness. Nevertheless, we use it all the time to test the state of consciousness or awareness of others, creating test stimuli too weak to attract attention but able to arouse a perceptible reaction in an attentive mind (whispering, for instance, the question Are you awake?).

Any definition of coherence based on causal connectedness within the mind and with the environment must rely on the introduction of events that could not have been expected. Otherwise any "effect" could be the result of anticipation or of stored patterns rather than a causal effect of the observed event. It is therefore important to make the distinction between logical and historical processes. In a logical process all events can be foreseen in principle. Simple examples are periodic processes and processes produced by play-back from a record. Others are those in which events can be anticipated on the basis of fixed sets of rules. A historic process is one that is dominated by unforeseeable events. Only historic processes do attract our attention for any period of time. Our brain is very good at catching regularities and has memory mechanisms to store and retrieve them, in this way quickly trivializing logical processes by detecting their regularity.

Imagine a brain state in which the different modalities all ran down their own process, going through the same sequence of topics, each speaking its own language, but synchronized as a logical process like clockwork, not by interaction. (An approximation to this Gedankenexperiment may be the stereotyped performance of an over-learned piece of music.) We probably wouldn't admit this as a highly conscious process. We would rather be inclined to require an additional test, in which one of the modalities was made to trip, and unless the others adapted to re-establish coherence we wouldn't call the state conscious. By the same token, were we to discover that on the third planet of Sirius there was a civilization that had gone through the exact same stages of development like ours in perfect synchrony, we wouldn't conclude that one of them had been aware of the other all along, would rather insist on signs of causal connection before according that attribute. These would have to be based on random, unforeseen, events in one civilization and reactions to them in the other.

¿From the causal criterion for the state of consciousness it is clear that a snapshot of a brain or of a mind, taken with a super-cerebroscope, cannot tell us at all whether that organism was in a conscious state: we couldn't perform causal tests. Indeed, the argument makes clear that it is misleading to speak of a conscious state at all: we should always speak of a process to be observed over some stretch of time. The empirical test for causality requires the creation of sets of events that couldn't possibly be due to accidental coincidence or to 
relations pre-established at an earlier time (like Leibniz' "pre-stabilized harmony" between mind and body). Our method for doing so is to create events that could not possibly have been predicted (in their timing, for instance) and to test whether they have consistent consequences.

Thus, consciousness cannot be conceived as a static configuration but only as a historic process in time. This, then, leads to another Gedanken-experiment: Use the supercerebroscope to record the brain process over a whole interval of time and store the result on a magnetic tape: Would that tape (or a "slave brain" controlled in detail by it) be conscious? To make the question more pointed, assume the recorded period contained clear examples of events that evidently were unexpected by the brain, and the record showed that each and every time all the different agents had reacted appropriately. I think we are all inclined to say that the recorded brain process was a conscious one, but that nevertheless the tape iteslf wasn't conscious because it certainly could not react to stimuli thrown at it: the replay of the tape would be a logical, not a historical process.

Subconscious States (and their Role in Creativity).

Subconscious states can be interpreted as those in which many of the agents in the brain are not functional or are not coherent with each other. Dreams, for instance, can be understood in many of their aspects as the result of temporary dysfunction in important modalities. In a dream I may imagine events as real - I fly, or I meet a person actually deceased, or I walk through a door in my house into a non-existent room - that my waking brain would perhaps allow me to imagine, but not without telling me at the same time that they were unreal and shouldn't be taken as basis for action. So in my dream the machinery for handling the reality status of an event - real and present, or real and past, or future but quite possible, or pure fancy, etc. - is not functional. For a discussion of subconscious, hypnotic states as states of reduced communication between sub-systems see (Hilgard 1977).

Subconscious activity may go on in our mind while we are consciously engaged in another matter. We usually find out about such activity in indirect ways, by discovering effects that cannot be attributed to mind processes that we remember, or if we remember them, cannot be traced back to a reason or purpose or to an act of will. Our basis for classifying mind processes as subconscious is the judgment that one or several important modalities have not contributed properly to the process.

Apparently, the lack of functionality of modalities, or their lack of coordination, doesn't correspond to sheer lack of neural activity. In fact, electro-encephalograms and other signals recorded from the healthy brain suggest that the whole brain is always active. The lack of mutual engagement of modalities or the total absence of some of them as active agents in our mind is probably rather due to the lack of correspondence or coordination or resonance, and therefore proper interaction, between sub-processes. A completely unconscious state of mind, in this image, is one in which there is a very low level of coherence between subsystems, to the extent that one cannot talk of a functional state at all, the brain not capable of reacting to any event altogether. 
A thought can establish itself in the mind only if none of the participating modalities throws in its veto. In a fully conscious state this is a very restrictive condition. Since among the modalities there are some that tell us how similar situations have been handled in the past, the conscious state has a tendency to restrict us to trodden paths. Disengaging modalities from a process liberates it from constraints and gives it more freedom to be creative (but also to err, of course). Poincaré's (1954) description of creative processes is very instructive: Work at the problem intensely and consciously (and just bear the frustration of not being able to solve it right away). Then do something else: engage your conscious mind in other activities and let your subconscious silently do its work. When you come back to the problem you may find that all of a sudden the pieces fall into place and you see the solution in a flash. This account easily jibes with the coherence definition of consciousness: While many of the usual modalities are out, being engaged in everyday activities, some idle agents are on their own, engage in play among themselves in ways that would never be permitted by some others, and before those come back they have found and locked in patterns that are novel and crucial to the solution sought.

Minimal Requirements for Consciousness.

It would be a ridiculous proposition to expect a system of coupled cavities, which I used as a physical metaphor, to be conscious as soon as it is pervaded by intimate causal connections. The difference to the brain must lie in the nature of the modalities and the relationships among them and with the environment. The essential property of the brain and its modalities is appropriate perceptual and motor coupling to the environment: the agents of the brain are there to organize behavior. In order to be able to do so any significant change in the environment must be appropriately reflected in the brain. This condition is a direct continuation of the coherence theory of consciousness and establishes the connection between consciousness and biological significance.

When speaking of the set of modalities required for a conscious mind one might consider a concept of simple additiveness, each sensory modality contributing just one more facet to the model of the world created internally. The collection of modalities that we require would then just be an accident of our own evolutionary history. We happen to have a certain complement of modalities and customarily require all of them to be involved in a mental state to be called conscious. If we were dolphins we would also ask for echo-acoustic processes to be part of a conscious thought. Turning this argument around, the debate about the existence of consciousness in a monkey or dog would be more of a quantitative issue than a qualitative one.

Brains may differ along several dimensions. One is the resolution of the raster with which modalities describe the world, the number of fibers in the optic or auditory nerves or the number of muscle fibers that can be controlled independently, for instance. An important difference between a mouse and a monkey is certainly one of resolution. Another dimension is extra perceptual modalities, such as color vision or echo-location. Still another is complements of functional schemata with which to structure the environment. Examples relevant to man are the usage of tools, long-range planning, language, or cultural activities such as music or mathematics. 
All of these dimensions add to the volume of consciousness and make it richer, or functionally more effective. But let's pose the question whether there is a clear-cut threshold below which we cannot possibly speak of consciousness, or whether it will always remain a matter of taste where we stop talking about consciousness, or a matter of just gradual reduction, when going down the ladder of complexity in the animal kingdom.

The decisive issue is whether the organism can couple to the environment in a meaningful way, whether it can successfully react to external events, whether it can survive in a historical world (and not just a logical world, for which an automaton may be fit). A minimal arrangement must certainly include two modalities, a sensory and a motor one, plus an adaptive translation mechanism between the two. To be sure, the two are required already for the more trivial reason that the organism has to be able to sense trouble and to move to avoid it. But beyond that the coupling of sensation and motility seem to be required for the establishment of functional coherence with the environment, a purely perceptual organism never being able to create a sensible reflection of the environment. It has been argued (Dru et al. 1975; Held and Hein 1963) that in order for learning to occur there has to be a motor system to actively create sensory events, that passive learning is not possible.

The situation relevant for the appearance of consciousness, then, is an environment characterized by a historical process and an organism with sensory and motor modalities between which translation is a non-trivial matter. We want this translation to be a non-trivial problem because otherwise we would call the organism an automaton, a purely logical entity (like an protozoan swimming up a chemical gradient). And the translation has to be nontrivial for the organism to establish coherence with the invironment: the only source of reliable knowledge is the establishment of highly non-trivial and therefore highly significant relationships between modalities.

It may be that coherence between modalities and with the environment can only be established with the help of objective representations. When my finger touches an object in front of me, my eye is able with great precision to predict the moment when my sense of touch will record the actual contact. This agreement can only be reached reliably if the participating modalities, vision, kinesthesia and touch, separately have a correct representation of the actual situation. Similarly, translation of Chinese into English (taken as two modalities) is not possible as a logical process without reference to a representation of the subject matter spoken about. Representation and meaning (Mephisto's "Schein des Himmelslichts" in Goethe's Faust), so much taken as the very essence of consciousness, would therefore be a functional must and not a superfluous luxury.

The representation a brain possesses of its environment is not a static entity, perhaps something like a description to some degree of precision, akin to a photograph, to which the brain converged after some time of exploration and learning (a view criticized in Freeman and Skarda 1990). We know an environment only relative to the activities we have organized in it. A tool with which we are intimately familiar will, for instance, take on a totally new life if we want to depict it in a drawing. The combinatorics of scenes and functional aspects is inexhaustible. When I speak of representation, then, I have to speak 
as much of the brain's potential to establish new objective properties of the environment relative to new activities as of the knowledge accumulated in past activities.

Turning to the simple end of the scale of animals, does a fly have its own low level of consciousness? After all, even a fly has modalities that can be in coherent functional states. To me the issue boils down to asking whether the fly is a logical device, an automaton, or whether it can flexibly react to new situations and create its own primitive representation of environmental facts. I wouldn't be surprised if it could.

What about machines? An automaton has a fixed (though possibly large) set of reaction patterns all of which are premeditated by the designer: an automaton is a logical and not a historical animal. It cannot create representations of the world (although the designer may put some of his in the machine). Any talk about machines that translate natural languages (Searle 1980) belongs to the realm of science fiction. No existing automaton displays any faculty to put us in doubt whether it had consciousness. The situation will be fundamentally changed once we understand the principle of autonomously learning organisms (as discussed in von der Malsburg 1985). That this principle can certainly be formulated algorithmically itself is as irrelevant as the fact that a thermodynamic system can be simulated on a computer - nobody takes it as an automaton for that reason.

Conclusion.

Unfortunately, my definition of consciousness as a state of high coherence among modalities has to rely intensely on implicit features of the environment and of the working brain itself. Compare this to the delightful explicitness of formal systems and universal Turing machines! My definition is not constructive: there is a proof of existence in the working brain, but before we can perform the test of building experimental organisms endowed with consciousness we will have to understand that brain much better.

Some authors discuss consciousness as if it was pure luxury, a largely superfluous feature over and above the biological function of the brain. If my definition does indeed address the essence of the phenomenon, then consciousness and proper function of the brain are just two sides of the same coin.

Much of the confusion that sometimes dominates discussions of consciousness arises because different people take very different aspects of our mind as the essence of the matter. One reaction to this could be that the definition of consciousness is an ill-defined issue, or a personal one, or both. I am, to the contrary, convinced that consciousness is fundamentally a rather well-defined phenomenon.

I take it as a reassuring sign that the aspect of inter-agent communication posited in my definition of consciousness is well reflected in traditional terminology. The Latin roots of the word "conscious" mean "being privy to," "sharing knowledge." The words "attention" or "to attend to" seem to derive their meaning from the image of a person orienting herself towards a thing or matter, getting ready to react to it. In the English language there are in addition the words "awareness" and "being aware of," which are translated in many languages synonymously with "consciousness" and "conscious of," although they 
are slightly more specialized to processes of perception. We use all of these expressions also when speaking of inter-human relations, as when I ask you, for instance, whether you are aware that we have a lunch appointment later today, meaning: do you know of it and are ready to take the necessary action. This usage is simply to be applied to the mind itself as if it was composed of different persons interacting with each other - in the example just given: "Is the memory and action-planning part of your brain coherent with the past lunch- appointment event in the linguistic modality of it?" Here as in so many cases expressions of common parlance contain very deep insight, a fact of which philosophy has profited so extensively.

With my basic stance - that consciousness doesn't reside in any specialized faculty of mind or localized structure of the brain, but is rather a cooperative phenomenon of the whole brain or mind - I am far from being alone. It is highly recommendable to read in this context a discussion of the problem by the famous neuropsychologist Luria (1978), which may be summed up in the citation "The cerebral basis of man's complex, semantic, system-based conscious activity must be sought in the combined activity of discrete brain systems, each of which makes its own special contribution to the work of the functional system as a whole." (p. 31). The electrophysiologist E. Roy John (1976) expresses similar opinions: "I believe that 'mind,' under which rubric are subsumed such phenomena as consciousness, subjective experience, the concept of self, and self-awareness, is an emergent property of sufficiently complex and appropriately organized matter." (p.1). "The content of consciousness is the sum of all informational processes in all the various functional systems of the brain. The information in each area comprises a coherent temporal pattern." (p. 39). Many others could be cited.

Although I cannot easily see a way to prove it, I find it conceivable that the qualia issue may be solved by stating that the quality of the taste of strawberries or of the color red or of the pain when my toe hits an obstacle is identical with all the processes and reactions that the sensation provokes or could provoke in my mind. (Maybe the most puzzling examples are those where the rational mind, represented by language, is most incapable of expressing those reactions.) So the color red may be gone for me when the color area of my brain is destroyed (accepting for the moment that claim in the literature), but that doesn't prove the essence of color resides in that area. You still need the whole brain to get to that essence, although all those reflection patterns aroused from the color area may vanish forever with its destruction. The point is illuminated to some extent by a metaphor. When trying to grasp the meaning of money it doesn't help to stare at a dollar bill. The bill by itself has little value. It derives its significance entirely from the patterns of beliefs and habits of people out there: hand it to a merchant and he is ready to give you something for it, his reaction depending in turn entirely on his expectation what others would sell for the bill or how much money they would be ready to pay for the object. Similarly it doesn't help staring at the neuron whose signal is linked with some elementary sensation - the essence of that sensation is in the reaction patterns of the whole brain (and body!).

If my discussion of consciousness is of any validity, the issue can be seen to run very much against the grain of science as we know it. Science has always had its great triumphs when and where it succeeded in subdividing complex phenomena into very simple paradigms. 
Doing the same to the brain we are in danger of being left with bits and pieces in our hand. Using the simile of just now, in order to understand the value of money, we shouldn't stare at dollar bills, but rather try to understand the system of beliefs and habits that give money its power. By the same token, none of the isolated components of the brain can be expected to hold the essence of consciousness. That is rather in the modes of interaction of all parts of the brain, and maybe even in the way the brain is integrated into the social world and the world at large. In this sense science may never understand consciousness in its substance. Nevertheless, it may still be a subject for good science, finding, for instance, ways to measure the level of consciousness of brain states. One such approach could just concentrate on statistical properties of signals exchanged between different modalities, or signal correlations between them, as a measure of the extent to which they are functionally coupled. Nature may, after all, have been friendly enough to provide us with an easily picked up signature of that state. Who knows, perhaps this signature is indeed nothing but a simple oscillatory signal, as advocated by Crick and Koch (1990).

\section{References.}

Crick, F. and Koch, C. (1990) Towards a neurobiological theory of consciousness. Seminars in the Neurosciences 2: 263-275.

Descartes, R. (1662) Traité de l'Homme, Paris.

Dru, D., Walker, J.P. and Walker, J.B (1975) Self-produced locomotion restores visual capacity after striate lesions. Science 187: 265-266.

Erdelyi, M.H. (1974) A new look at the new look: perceptual defense and vigilance. Psychol Rev. 81: 1-25.

Freeman, W.J. and Skarda, C.A. (1990) Representations: Who Needs Them? In: McGaugh, J.L., Weinberger, N.M. and Lynch, G. (eds.) Brain, Organization and Memory: Cells, Systems, and Circuits, pp. 375-380. Oxford University Press, New York, Oxford.

Held, R. and Hein, A. (1963) Movement-produced stimulation in the development of visually guided behavior. J. Comp. Physiol. Psychol. 56: 872-876.

Hilgard, E.R. (1977) Divided Consciousness: Multiple Controls in Human Thought and Action. Wiley, New York.

John, E.R. (1976) A Model of Consciousness. In: Schwartz, G.E. and Shapiro, D. (eds) Consciousness and Self-Regulation, pp. 1-50. John Wiley \& Sons, Chichester, New York.

Kahneman, D. (1973) Attention and Effort. Prentice Hall, Englewood Cliffs, N.J.

Luria, A.R. (1978) The Human Brain and Conscious Activity. In: Schwartz, G.E. and Shapiro, D. (eds.). Consciousness and Self-Regulation, Vol. 2, pp. 1-36. John Wiley \& Sons, Chichester, New York.

Mandler, G. (1975) Mind and Emotion. Wiley, New York. 
Minsky, M. (1988) The Society of Mind. Simon and Schuster, New York, London.

Poincaré, H. (1954) cited in: Ghiselin and Brewster (eds.) The Creative Process, pp. 22-31. Univ. of California Press, Berkeley.

Posner, M.I. (1987) Chronometric Explorations of Mind. Erlbaum, Hillsdale, NJ.

Searle, J.R. (1980) Minds, brains, and programs. Behavioral and Brain Sciences 3: 417458.

Shallice, T. (1978) The dominant action system: an information processing approach to consciousness. In: Pope, K.S., Singer, J.L. (eds) The Stream of Consciousness: Scientific Investigations into the Flow of Human Experience, pp. 117-157. Plenum, New York, London.

Sperry, R.W. (1966) Brain bisection and consciousness. In: Eccles, J.(ed), Brain and Conscious Experience, p. 298. Springer, Berlin, New York.

Toulouse, G., Dehaene, S. and Changeux, J.-P. (1986) A spin glass model of learning by selection. PNAS 83: 1695.

von der Malsburg, C., (1985) Algorithms, Brain, and Organization. In: Demongeot, J., Golès, E., Tchuente, M. (eds) Dynamical Systems and Cellular Automata, pp. 235-246. Academic Press, London. 